\title{
Exact Coarse Graining Preserves Entropy Production out of Equilibrium
}

\author{
Gianluca Teza \\ Department of Physics of Complex Systems, Weizmann Institute of Science, Rehovot 7610001, Israel and \\ Department of Physics and Astronomy, University of Padova, Via Marzolo 8, I-35131 Padova, Italy \\ Attilio L. Stella* \\ Department of Physics and Astronomy, University of Padova, Via Marzolo 8, I-35131 Padova, Italy and \\ INFN, Sezione di Padova, Via Marzolo 8, I-35131 Padova, Italy
}

(Dated: November 25, 2020)

\begin{abstract}
The entropy production rate associated with broken time-reversal symmetry provides an essential characterization of nanosystems out of equilibrium, from driven colloidal particles to molecular motors. Limited access to the dynamical states is generally expected to hinder the correct estimation of this observable. Here we show how memoryless jump processes can be coarse grained exactly preserving its average and fluctuations at stationarity. This supports univocal applicability of fluctuation theorems for entropy and allows inference of the genuine thermodynamics together with inaccessible process details.
\end{abstract}

Entropy production out of equilibrium, measured, e.g., from the heat dissipated by a mesoscopic system into a thermostat, is a key to interpret experiments involving nanomanipulation or molecular motors [1 6]. When dealing with active matter, this production may be the only possible indicator of out of equilibrium conditions [7, 8]. Fluctuations of the rate of produced entropy are expected to obey symmetry properties that allow us to estimate free energy differences or binding energies at the molecular level [1, 9 11]. Theorems validating such properties have been proved for specific models [12 15], but do not hold if only part of the states of the mesoscopic system is experimentally accessible. Indeed, applicability of these theorems requires that all slow transitions between mesostates are detectable, and that transitions inside each mesostate are very fast [16, 17]. Under these conditions entropy production can be recovered from a description without memory in the framework of stochastic thermodynamics [18.

On the other hand, in experiments where not all mesoscopic details are accessible, like with molecular motors, nonexponential dwelling or residence time distributions have been often measured. 19 21. These memory effects were identified as revealing features of the underlying chemomechanical transitions, but not put in direct relation to entropy production.

If only partial, coarse-grained information is experimentally available, the average entropy production one can record is generally expected to be lower [17]. Estimates of partial entropy production pertaining to the accessible parts of the systems, or lower bounds for the average full productions, have been actively studied recently [7, 8, 22, 26]. However, these results are of limited help for a complete thermodynamic inference [27], since precise insight on the possible effects of coarse graining on detectable entropy production is still missing [17].

\footnotetext{
* attilio.stella@pd.infn.it
}

The entropy production of coarse-grained Markov processes governed by Master equations was first studied in cases in which microstates connected by fast transitions rates were lumped into slow evolving mesostates, implying a sharp timescale separation [28]. So, for large separation the memory effects could be regarded as a small perturbation. More recently, it has been shown 29, 30. that even in the limit of infinite timescale separation the resulting effective slow Markov dynamics generally fails to account for the whole original production. Thus, establishing the precise effects of coarse graining on the detectable entropy production and finding if there exists one way to coarse grain leaving such production invariant remains an open fundamental issue, of much relevance for the description of experiments.

In this Letter we present an exact coarse graining by decimation of Markov jump processes which keeps precise record of how the entropy production evolves in time. We also show that its average and higher fluctuation moments remain invariant at stationarity. Non-Markovian residence time distributions typically recorded in experiments can also be analyzed in terms of a complementary form of decimation of Markovian trajectories in state space. The results of this second decimation do not generally allow us to recover the full entropy production, but can enable its thermodynamic inference [17, 27] by reconstruction of the underlying dynamics.

We start by considering a Markov process on a linear periodic network with states $i=1,2, \ldots, N$ and rates of jump $W_{j i}$ from state $i$ to state $j$. We assume nonzero rates only for nearest neighbor jumps, and put $W_{j i}=$ $r$ or $W_{j i}=l$, for all right or left jumps, respectively [Fig. 1(a)]. If states, e.g., refer to positions of a particle on a lattice with spacing $L, r$ and $l$ can be linked to $L$, to a uniform driving force $f$, and to the thermal bath temperature $T$, by the local detailed balance condition $r / l=e^{f L / k_{B} T}[31$. Along a trajectory of the process in which jumps take place at times $t_{k}(k=1,2, \ldots n$; $\left.0<t_{1}<t_{2}<\ldots t_{n}<t\right)$ from states $i_{k-1}$ to states $i_{k}\left(i_{0}\right.$ 


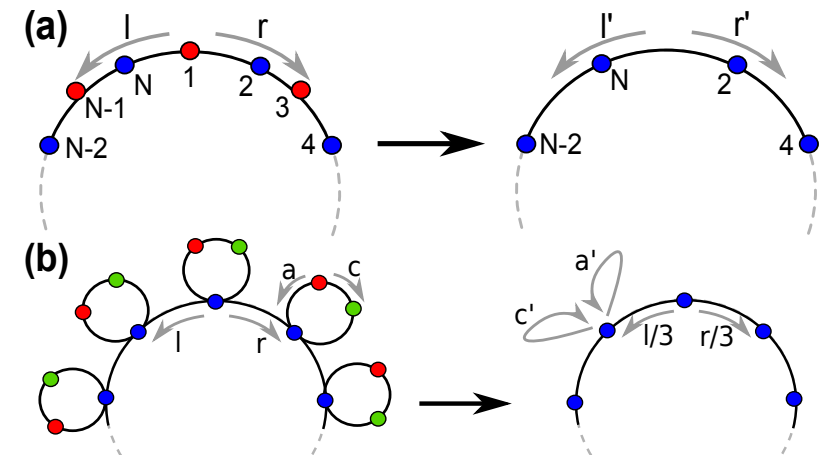

FIG. 1. (a) Linear network of Eq. 2. decimation yields the process of Eq. 6] with jumping rates $r^{\prime} \propto r^{2}$ and $l^{\prime} \propto l^{2}$. (b) Chain with secondary loops of Eq. 8 decimation leaves a linear chain in which every site has additional entropy producing self jumps with rates $a^{\prime} \propto a^{3}$ and $c^{\prime} \propto c^{3}$.

state at $t=0)$ the entropy produced is $\left(k_{B}=1\right)[15,32$,

$$
S=\sum_{k=1}^{n} \log \left[\frac{W_{i_{k}, i_{k-1}}}{W_{i_{k-1}, i_{k}}}\right],
$$

with average rate at stationarity compensating exactly the entropy transfered to the medium.

The probability $\bar{P}_{i}(t)$ for the system to be in state $i$ at time $t$, is marginal of the probability $P_{i}(S, t)$ that the system is in state $i$ at time $t$ with an entropy $S$ accumulated along all possible trajectories. Thus we can write:

$$
\begin{aligned}
{\left[r+l+\partial_{t}\right] P_{i}(S, t) } & =r P_{i-1}(S-\log (r / l), t)+ \\
& +l P_{i+1}(S+\log (r / l), t)
\end{aligned}
$$

where the shifts in the $S$ arguments on the right-hand side account for the entropy gains associated with jumps according to Eq. 1 . Summing Eq. 2 over all $S$ values yields the master equation satisfied by $\bar{P}_{i}(t)$ with a unique stationary solution reached for $t \rightarrow \infty$ from arbitrary initial conditions 33. For $t \rightarrow \infty, P_{i}(S, t)$ is consistent with a large deviation principle [34] for the entropy production rate $\sigma=S / t$. Indeed, indicating by $Q(S, t)=\Sigma_{i} P_{i}(S, t)$ the probability of having produced a total entropy $S$ at time $t$, the scaled cumulant generating function (SCGF) $\varepsilon$ can be extracted from the function $G(\lambda, t)=\sum_{S} e^{\lambda S} Q(S, t) \sim_{t \rightarrow \infty} e^{\varepsilon(\lambda, r, l) t}[34$. By discrete Laplace-transforming Eq. 2 with respect to the entropy $S$ we obtain a first order differential equation for $G$

$$
\left[\partial_{t}+r+l+r e^{\lambda \log r / l}+l e^{\lambda \log l / r}\right] G(\lambda, t)=0
$$

whose solution, in the long time limit, provides us with the SCGF 35.

$$
\varepsilon=r e^{\lambda \log r / l}+l e^{-\lambda \log (r / l)}-(r+l) .
$$

The probability $Q(S, t)$ for $t \rightarrow \infty$ concentrates on the value $S=\sigma_{0} t$ where $\sigma_{0}=\partial \varepsilon /\left.\partial \lambda\right|_{\lambda=0}=(r-l) \log (r / l)$, while higher order derivatives $\partial^{n} \varepsilon /\left.\partial \lambda^{n}\right|_{\lambda=0}$ give the scaled cumulants of $\sigma$ describing its fluctuations for long times. Since $\varepsilon$ satisfies the fluctuation theorem [15, i.e. $\varepsilon(\lambda-1, r, l)=\varepsilon(-\lambda, r, l)$, the probability of $\sigma$ further satisfies $\operatorname{Pr}(S / t=\sigma) / \operatorname{Pr}(S / t=-\sigma)=e^{\sigma t}$ for $t \rightarrow \infty$.

Our coarse graining based on algebraic elimination of probabilities from equations like Eq. 2, can, in principle, be applied for arbitrary choices of the surviving states. The linear character of the network in Eq. 2 suggests a choice reducing to a minimum the complexity of the calculations and preserving the homogeneity of the model. Indeed, assuming $N>3$ even, one can eliminate from the system in Eq. 2 all odd (or even) states [Fig. 1(a)]. So, after Fourier transforming in time Eq. 2 one obtains

$$
\begin{aligned}
{[r+l+i \omega] \tilde{P}_{i}(S, \omega)=} & r \tilde{P}_{i-1}(S-\log (r / l), \omega)+ \\
& +l \tilde{P}_{i+1}(S+\log (r / l), \omega)
\end{aligned}
$$

where $\tilde{P}_{i}(S, \omega)=\int_{\mathbb{R}} d t e^{i \omega t} P_{i}(S, t)$, and the odd $\tilde{P}_{i}$ 's can be algebraically eliminated. Thus, upon reverse transforming, the even $P_{i}$ 's satisfy

$$
\begin{gathered}
{\left[\frac{1}{2(r+l)} \partial_{t}^{2}+\partial_{t}\right] P_{i}(S, t)=\frac{r^{2}}{2(r+l)} P_{i-2}(S-2 \log (r / l), t)+} \\
\quad+\frac{l^{2}}{2(r+l)} P_{i+2}(S+2 \log (r / l), t)-\frac{r^{2}+l^{2}}{2(r+l)} P_{i}(S, t) .
\end{gathered}
$$

Equations 6 are not consistent with a master equation due to the $\partial_{t}^{2}$ term. The quantity $\sum_{S} \sum_{i \text { even }} P_{i}(S, t)$ is not conserved, but stabilizes at a value $1 / 2$ (equivalence of even and odd states) after a transient time $1 / 2(r+l)$. As we show below, in spite of the lack of strict normalization of the $P_{i}$ 's with even $i$, the coarsegrained description provided by Eqs. 6 accounts correctly for the entropy production of the original system. Indeed, we can define $Q^{\prime}(S, t)=\sum_{i \text { even }} P_{i}(S, t)$ and write a differential equation of second order in time for $G^{\prime}(\lambda, t)=\sum_{S} e^{\lambda S} Q^{\prime}(S, t)$, which becomes the function controlling entropy production in the coarse-grained system:

$$
\begin{aligned}
& {\left[\partial_{t}^{2}+2(r+l) \partial_{t}+r^{2}+l^{2}\right] G^{\prime}(\lambda, t)=} \\
& =\left[r^{2} e^{2 \lambda \log (r / l)}+l^{2} e^{-2 \lambda \log (r / l)}\right] G^{\prime}(\lambda, t) .
\end{aligned}
$$

From the dominant long $t$ behavior $G^{\prime} \sim e^{\varepsilon^{\prime} t}$ of the solution, we argue that the rate $S / t$ still obeys a large deviation principle 34 and find eventually the SCGF for the coarse-grained entropy production $\varepsilon^{\prime}(\lambda, r, l)=$ $\varepsilon(\lambda, r, l)[35$. Thus, the SCGF function remains the same as that of the original process satisfying the fluctuation theorem [15]. This is a first instance of our main, unexpected 17, 28, 36 result: this decimation leaves exactly invariant the stationary spectrum of entropy production fluctuations.

Memory effects in Eqs. 6are represented by the higherorder time derivative terms and each even $\bar{P}_{i}(t)$ has nonzero contributions only from the trajectories that at time $t$ see state $i$ occupied, while trajectories visiting odd states at the same time do not contribute to any $\bar{P}_{i}(t)$. 
There is a complementary way of decimating the trajectories in which memory reveals in nonexponential probability densities of jump times between surviving states. In this second form of decimation also trajectories which see odd states occupied at time $t$ contribute to the probability of occupation of the even states. Indeed, one can ascribe to occupation of an even state $i$ also the time intervals during which state $i-1$ or $i+1$ are visited, up to when state $i+2$ or $i-2$ are first reached [35. In this way the probability of occupation of the surviving states remains strictly normalized to 1 . Probability densities of such and related times are often recorded in experiments [1921], and to compute them we introduce a normalized jump rate $w_{j i}=W_{j i} / \sum_{k} W_{k i}$. So, for the Markov process, the probability density of the time $t$ of jump from state $i$ to state $j$ after arriving in $i$ at $t=0$ has the exponential form $w_{j i} e^{-t / \tau} / \tau=\frac{1}{2 \pi} \int \mathrm{d} \omega e^{-i \omega t} \tilde{\pi}_{j i}(\omega)$, with $\tau=(r+l)^{-1}$. The Fourier transform $\tilde{\pi}_{j i}$ allows to express as a series of convolutions the characteristic function of the probability density $p_{r}(t)$ of jump times from an even state $i$ to the state $i+2$ without visiting $i-2$. To this purpose we consider the $3 \times 3$ matrix $T_{k^{\prime} k}(\omega)=\tilde{\pi}_{k^{\prime} k}(\omega)$, restricted to the states $\{i-1, i, i+1\}$. In this way the characteristic function of $p_{r}(t)$ can be written as $\tilde{p}_{r}(\omega)=\int \mathrm{d} t e^{i \omega t} p_{r}(t)=\tilde{\pi}_{i+2, i+1}(\omega)(\mathbb{I}+\mathbf{T}(\omega))_{i+1, i}^{-1}$. This expression sums the contributions of all trajectories performing that jump [35]. Analogously one obtains $\tilde{p}_{l}(\omega)$. Unlike in Eqs. 6, where the $P_{i}$ 's are constructed by recording the presence of the system exclusively in the even states, one can count the whole times of jump as residence times in state $i$. With this assumption the sum $p_{r}(t)+p_{l}(t)=p(t)$ becomes equivalent to a non-Markovian (nonexponential) distribution of residence time in a generic even state. This distribution is reported in Fig. 2a. The process resulting from this interpretation of the jump times qualifies as semi-Markov with time-direction independence, since $p_{r}(t) \propto p_{l}(t)$ 37 39. The entropy production rate of such models has been determined recently [38, 39] and is remarkably consistent with our results for the linear chain 35. As we show below, in more general cases such consistency does not hold. However, the densities $p_{r, l}(t)$, regarded as empirical data, can be exploited to identify the underlying Markov dynamics. Upon matching these densities with analytic results like those just derived, one can determine the rates of the undecimated model and infer the full entropy production with its fluctuations. In relatively simple cases a successful identification could be guided by physical and analytical insight. At general level establishing conditions under which it would be possible and unique poses a future challenge.

The linear network of Eq. 2 presents only one loop along which entropy is produced. A fundamental problem left is to study situations in which coarse graining erases loops producing entropy in the network. Indeed, detectable entropy production is generally expected to lack erased loop contributions and therefore to be lower in such cases [17, 36].

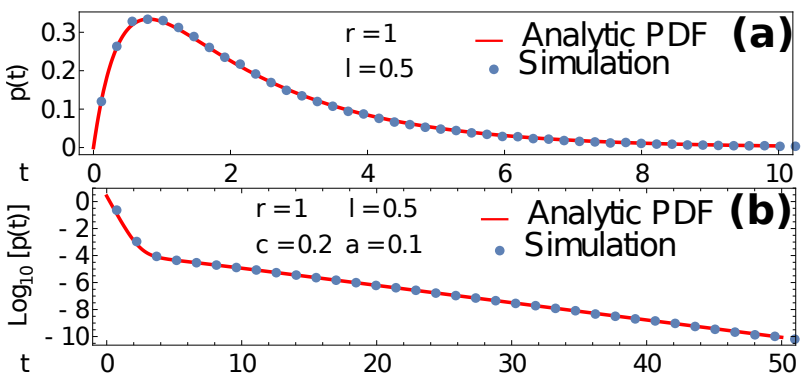

FIG. 2. Residence time distributions from decimation of the systems in Eqs. 2 (a) and Eqs. 8 (b): analytic results (red lines) and data (blue dots) from decimation of trajectories simulated with the Gillespie algorithm [35, 40].

Let us consider the network in Fig. 1b. It consists of a main loop of $N$ states (blue), $X_{1}, X_{2}, \ldots, X_{N}$, with right $\backslash$ left nearest neighbor jump rates $r \backslash l$. A secondary 3 -state loop is further attached to each $X_{i}$ state along the main loop, by connecting it to a $Y_{i}$ (red) and a $Z_{i}$ (green) state. Rates $c$ and $a$ apply, respectively, to clockwise and anticlockwise jumps to nearest neighbors within secondary loops. The states $Y_{i}$ and $Z_{i}$ of each loop are those we want to decimate. Before decimation, upon summing over the index $i$ specifying different $X, Y$ and $Z$ states equations analogous to Eq. 2 [35], one gets:

$$
\left\{\begin{aligned}
\partial_{t} P_{X}(S, t) & =r P_{X}\left(S-\log \frac{r}{l}, t\right)+l P_{X}\left(S-\log \frac{l}{r}, t\right)+ \\
& +c P_{Y}\left(S-\log \frac{c}{a}, t\right)+a P_{Z}\left(S-\log \frac{a}{c}, t\right)+ \\
& -[r+l+a+c] P_{X}(S, t) \\
\partial_{t} P_{Y}(S, t) & =a P_{X}\left(S-\log \frac{a}{c}, t\right)+c P_{Z}\left(S-\log \frac{c}{a}, t\right)+ \\
& -[a+c] P_{Y}(S, t) \\
\partial_{t} P_{Z}(S, t) & =c P_{X}\left(S-\log \frac{c}{a}, t\right)+a P_{Z}\left(S-\log \frac{a}{c}, t\right)+ \\
& -[a+c] P_{Z}(S, t)
\end{aligned}\right.
$$

where, e.g., $P_{X}(S, t)=\Sigma_{i} P_{X_{i}}(S, t)$ is the probability that a trajectory ends at time $t$ in a generic $X$ state with cumulated entropy $S$. The SCGF $\varepsilon(\lambda, r, l, c, a)$ for entropy production of this process can be found by considering, for $\alpha=X, Y, Z, G_{\alpha}(\lambda, r, l, a, c)=\sum_{S} e^{\lambda S} P_{\alpha}(S, t)$ in Eqs. 8 and by diagonalizing the $3 \times 3$ matrix expressing the time derivative of the $G_{\alpha}$ vector components. The dominant eigenvalue determines $\varepsilon$ [35] which yields an average entropy production rate:

$$
\sigma_{0}=\left.\frac{\partial \varepsilon}{\partial \lambda}\right|_{\lambda=0}=\frac{r-l}{3} \log \frac{r}{l}+(c-a) \log \frac{c}{a} .
$$

Here the first term is the contribution from jumps on the main loop, while the latter is relative to transitions occurring within the secondary loops. Decimation in this case is realized by simply eliminating $P_{Y}(S, t)$ and $P_{Z}(S, t)$ from the system of Eqs. 8 after Fourier transforming in time. Reverse transforming yields a third-order differential equation in time for $P_{X}(S, t)$. Also in this case $\sum_{S} P_{X}(S, t)$ is not strictly normalized, but for large $t$ it stabilizes to $1 / 3$. Indeed, $X, Y$ and $Z$ states have equal 
total probability $1 / 3$ at stationarity [35. $P_{X}(S, t)$ plays here a role analogous to that of $Q^{\prime}(S, t)$ in the linear chain decimation. For the function $G^{\prime}(\lambda, t)=\Sigma_{S} e^{\lambda S} P_{X}(S, t)$ of the coarse-grained network we eventually obtain

$$
\left[\alpha+\beta \partial_{t}+\gamma \partial_{t}^{2}+\partial_{t}^{3}\right] G^{\prime}(\lambda, t)=0
$$

where $\alpha, \beta$ and $\gamma$ are functions of the jump rates and of $\lambda$ 35. In the limit $t \rightarrow \infty$ we get $G^{\prime} \sim e^{t \varepsilon^{\prime}}$, where $\varepsilon^{\prime}$ is the dominant root of the characteristic equation associated with Eq. 10. Remarkably, $\varepsilon^{\prime}$ coincides with the $\mathrm{SCGF} \varepsilon$ of the original process 35. So, also in this case $\varepsilon^{\prime}=\varepsilon$, maintaining validity of the fluctuation theorem. In this example, the elimination of secondary loops leads to terms in Eq. 10 that represent additional entropy gains originating from self jumps on $X$ states in the coarse-grained dynamics [Fig. 1(b)]. Such contributions take into account what the removed secondary loops were producing in the underlying Markov description [35]. Thanks to them, contrary to previous expectations 17, 36, our decimation can keep track of the full entropy production.

Decimation of the process trajectories, carried on along lines similar to those illustrated for the linear chain, allows us to obtain nonexponential $p_{r}(t)$ and $p_{l}(t)$ for the jumps between neighboring $X$ states [35. Also in this case the coarse-grained dynamics can be regarded as semi-Markov with time-direction independence $\left[p_{r}(t) \propto\right.$ $p_{l}(t)$. In Fig. 2(b) we compare the residence time distribution $p(t)=p_{r}(t)+p_{l}(t)$ with a histogram based on simulations.

Evaluating the rate of entropy production of this semiMarkov model according to Refs. [38, 39] would account only for the partial contribution coming from direct transitions between surviving $X$ states, with average $\log (r / l)(r-l) / 3<\sigma_{0}$. Also procedures setting lower bounds to the average entropy production rate [7] could not improve the estimate of this average, due to the timedirection independence of the process. However, even though coarse graining gives access only to the densities $p_{r}(t)$ and $p_{l}(t)$, the calculations leading to these functions still allow us to determine the full entropy production. This can be done by matching the empirical probability densities of jump times with those of the candidate model for the fine-grained process. Its Markovian dynamics can be precisely determined together with the full spectrum of entropy production. The marked difference between the residence time distributions reported in panel (a) and (b) of Fig. 2, exemplifies a useful hint helping in an attempt to guess the underlying hidden Markov network.

Our coarse graining can be applied, e.g., to molecular motors. A simplified version of the model in Ref. 5] is reported in Fig. 3. where the red dots in the periodic network indicate intermediate hidden $Y$ states allowing ATP hydrolysis activated jumps between the blue $X$ states. The positions of the $X$ states are recorded in experiments. The transition rates reported in Fig. 3 satisfy local detailed balance conditions linking them to the load force $f$, the difference in chemical potentials

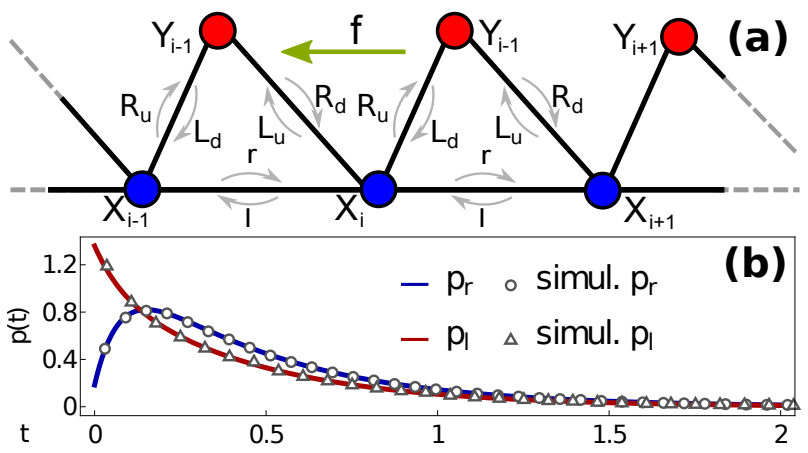

FIG. 3. (a) Markov jump molecular motor model. Matching $p_{r}(t)$ and $p_{l}(t)(\mathbf{b})$ allows us to recover all rates, full entropy production and ATP consumption.

$\Delta \mu=\mu(A T P)-\mu(A D P)-\mu(P)$, the temperature $T$ and the spacing $L$ between $X$ positions [35]. Elimination from the equations of the probabilities referring to $Y$ sates leads to the following system for the probabilities of the $X$ states

$$
\begin{aligned}
& {\left[\left(r+l+R_{d}+L_{u}+R_{u}+L_{d}\right) \partial_{t}+\partial_{t}^{2}\right] P_{X_{i}}(S, t)=} \\
& \quad=r\left(R_{d}+L_{d}\right) P_{X_{i-1}}(S-\log (r / l), t)+ \\
& \quad+l\left(R_{d}+L_{d}\right) P_{X_{i+1}}(S-\log (l / r), t)+ \\
& \quad+R_{d} R_{u} P_{X_{i-1}}\left(S-\log \left(R_{d} R_{u} / L_{u} L_{d}\right), t\right)+ \\
& \quad+L_{u} L_{d} P_{X_{i+1}}\left(S-\log \left(L_{u} L_{d} / R_{d} R_{u}\right), t\right)+ \\
& \quad-\left[(r+l)\left(R_{d}+L_{u}\right)+R_{d} R_{u}+L_{d} L_{u}\right] P_{X_{i}}(S, t)
\end{aligned}
$$

The various terms on the right-hand side account for the entropy contributions due to both direct and ATP activated transitions. Exact calculations show that this coarse-grained description accounts for the total entropy production also in this case [35]. The process resulting from analysis of the probability densities of jump times is semi-Markov with time-direction dependence, since $p_{r}(t)$ and $p_{l}(t)$, now reported in Fig. 3(b), are not simply proportional. The methods of Refs. [38, 39] do not allow to exploit this dependence for the computation of the entropy production of such process 7 . On the other hand, if the jump time probability densities are extracted from some empirical time series, one can recover the rates of the original Markov model by matching them with the results from a decimation of trajectories letting only $X$ states survive. So, provided the assumption of underlying Markov dynamics is correct, a successful matching allows us to recover the full entropy produced in the experiment.

The decimation of equations presented above can be performed on networks with arbitrary topology and with states surviving decimation that are not simply equivalent up to translations. Referring to the simple linear chain case, there is no problem in letting, for example, only a subset of odd or even states survive decimation, even if this breaks the original homogeneity of the transition rates.

An example with inhomogeneity allowing us to make 
contact with the timescale separation situations considered in Refs. 28 30, is that of a linear chain where nearest neighbor links having fast rates, $R$ and $L$, alternate with links having slow rates, $r$ and $l$. In the decimated equations only one state for each pair of two fast connected states survives to represent the whole cluster, and the coarse-grained dynamics again preserves entropy production for arbitrary timescale separations. This example is treated in detail in Ref. [35]. One can also keep simultaneous record of other currents besides the entropy production rate 41 .

Summarizing, we showed that, in the context of Markov jump processes, an exact coarse graining, taking into account memory effects, and guaranteeing invariance of average and fluctuations of the full entropy production at stationarity, is possible. While an extension of the type of exact results presented here to other models is an open program, our findings suggest that future investigations in this field, both theoretical and experimental, should focus on the memory effects associated with coarse graining, which revealed essential to guarantee entropy production invariance 22]. These effects, when regarded as a result of our decimation of trajecto- ries, can be also crucial for a thermodynamic inference strategy. Indeed, after detecting non-Markovian probability densities for the times of jump in an experimentally studied process, one can try to match them with those of a guessed underlying Markov process. If successful, this enables to determine the correct full entropy production, while uncovering hidden states and mechanisms. An assumption at the basis of this strategy is that Markov jump dynamics is adequate to describe what underlies the coarse-grained level. Such dynamics should, in principle, result from coarse-graining of a more microscopic description, and the choice of rates may reveal crucial for the very possibility of describing certain phenomena [42].

\section{ACKNOWLEDGMENTS}

We acknowledge Carlo Vanderzande, Marco Baiesi and Stefano Iubini for collaboration on related subjects. We thank David Mukamel and Oren Raz for discussions. G.T. is supported by a research grant from the Center of Scientific Excellence at the Weizmann Institute of Science and by the grant of Simons Foundation.
[1] J. Liphardt, S. Dumont, S. B. Smith, I. Tinoco, and C. Bustamante, Equilibrium information from nonequilibrium measurements in an experimental test of Jarzynski's equality., Science (New York, N.Y.) 296, 1832 (2002)

[2] G. M. Wang, E. M. Sevick, E. Mittag, D. J. Searles, and D. J. Evans, Experimental Demonstration of Violations of the Second Law of Thermodynamics for Small Systems and Short Time Scales, Physical Review Letters 89, 050601 (2002).

[3] C. Bustamante, J. Liphardt, and F. Ritort, The Nonequilibrium Thermodynamics of Small Systems, Physics Today 58, $43(2005)$

[4] A. B. Kolomeisky and M. E. Fisher, Molecular Motors: A Theorist's Perspective, Annual Review of Physical Chemistry 58, 675 (2007).

[5] A. W. C. Lau, D. Lacoste, and K. Mallick, Nonequilibrium Fluctuations and Mechanochemical Couplings of a Molecular Motor, Physical Review Letters 99, 158102 (2007).

[6] R. D. Astumian and P. Hänggi, Brownian Motors, Physics Today 55, 33 (2002).

[7] I. A. Martínez, G. Bisker, J. M. Horowitz, and J. M. Parrondo, Inferring broken detailed balance in the absence of observable currents, Nature Communications 10, 10.1038/s41467-019-11051-w (2019), arXiv:1809.02084.

[8] É. Roldán, J. Barral, P. Martin, J. M. R. Parrondo, and F. Jülicher, Arrow of Time in Active Fluctuations, (2018), arXiv:1803.04743

[9] G. Hummer and A. Szabo, Free energy reconstruction from nonequilibrium single-molecule pulling experiments, Proceedings of the National Academy of Sciences of the United States of America 98, 3658 (2001)

[10] A. Mossa, M. Manosas, N. Forns, J. M. Huguet, and
F. Ritort, Dynamic force spectroscopy of DNA hairpins: I. Force kinetics and free energy landscapes, Journal of Statistical Mechanics: Theory and Experiment 2009, 10.1088/1742-5468/2009/02/P02060 (2009), arXiv:0902.3632

[11] J. Camunas-Soler, A. Alemany, and F. Ritort, Experimental measurement of binding energy, selectivity, and allostery using fluctuation theorems, Science (New York, N.Y.) 355, 412 (2017)

[12] D. J. Evans, E. G. D. Cohen, and G. P. Morriss, Probability of second law violations in shearing steady states, Physical Review Letters 71, 2401 (1993).

[13] G. Gallavotti and E. G. D. Cohen, Dynamical Ensembles in Nonequilibrium Statistical Mechanics, Physical Review Letters 74, 2694 (1995).

[14] J. Kurchan, Fluctuation theorem for stochastic dynamics, Journal of Physics A: Mathematical and General 31, 3719 (1998)

[15] J. L. Lebowitz and H. Spohn, A Gallavotti-Cohen-Type Symmetry in the Large Deviation Functional for Stochastic Dynamics, Journal of Statistical Physics 95, 333 (1999)

[16] J. Mehl, B. Lander, C. Bechinger, V. Blickle, and U. Seifert, Role of Hidden Slow Degrees of Freedom in the Fluctuation Theorem, Physical Review Letters 108, 220601 (2012)

[17] U. Seifert, From Stochastic Thermodynamics to Thermodynamic Inference, Annual Review of Condensed Matter Physics 10, 171 (2019)

[18] U. Seifert, Stochastic thermodynamics, fluctuation theorems and molecular machines, Reports on Progress in Physics 75, 126001 (2012).

[19] M. Rief, R. S. Rock, A. D. Mehta, M. S. Mooseker, R. E. Cheney, and J. A. Spudich, Myosin-V stepping kinetics: 
A molecular model for processivity, Proceedings of the National Academy of Sciences of the United States of America 97, 9482 (2000)

[20] A. B. Kolomeisky and M. E. Fisher, Extended kinetic models with waiting-time distributions: Exact results, Journal of Chemical Physics 113, 10867 (2000).

[21] V. Bierbaum and R. Lipowsky, Dwell Time Distributions of the Molecular Motor Myosin V, PLoS ONE 8, 10.1371/journal.pone.0055366 (2013).

[22] A. Crisanti, A. Puglisi, and D. Villamaina, Nonequilibrium and information: The role of cross correlations, Physical Review E - Statistical, Nonlinear, and Soft Matter Physics 10.1103/PhysRevE.85.061127 (2012), arXiv:1202.0508.

[23] K. Kawaguchi and Y. Nakayama, Fluctuation theorem for hidden entropy production, Physical Review E - Statistical, Nonlinear, and Soft Matter Physics 88, 10.1103/PhysRevE.88.022147 (2013), arXiv:1209.6333.

[24] M. Polettini and M. Esposito, Effective Thermodynamics for a Marginal Observer, Physical Review Letters 119, 10.1103/PhysRevLett.119.240601 (2017), arXiv: 1703.05715 .

[25] G. Bisker, M. Polettini, T. R. Gingrich, and J. M. Horowitz, Hierarchical bounds on entropy production inferred from partial information, Journal of Statistical Mechanics: Theory and Experiment 2017, 10.1088/17425468/aa8c0d (2017), arXiv:1708.06769.

[26] J. Gladrow, N. Fakhri, F. MacKintosh, C. Schmidt, and C. Broedersz, Broken Detailed Balance of Filament Dynamics in Active Networks, Physical Review Letters 116, 248301 (2016)

[27] A. Alemany, M. Ribezzi-Crivellari, and F. Ritort, From free energy measurements to thermodynamic inference in nonequilibrium small systems, New Journal of Physics 17, 075009 (2015)

[28] S. Rahav and C. Jarzynski, Fluctuation relations and coarse-graining, Journal of Statistical Mechanics: Theory and Experiment 2007, P09012 (2007)

[29] M. Esposito, Stochastic thermodynamics under coarse graining, Physical Review E - Statistical, Nonlinear, and Soft Matter Physics 10.1103/PhysRevE.85.041125 (2012), arXiv:1112.5410.
[30] S. Bo and A. Celani, Entropy Production in Stochastic Systems with Fast and Slow Time-Scales, Journal of Statistical Physics 10.1007/s10955-014-0922-1 (2014).

[31] S. Katz, J. L. Lebowitz, and H. Spohn, Phase transitions in stationary nonequilibrium states of model lattice systems, Physical Review B 28, 1655 (1983).

[32] U. Seifert, Entropy Production along a Stochastic Trajectory and an Integral Fluctuation Theorem, Physical Review Letters 95, 040602 (2005).

[33] N. G. van. Kampen, Stochastic processes in physics and chemistry (North-Holland, 1992) p. 465.

[34] H. Touchette, The large deviation approach to statistical mechanics, Physics Reports 478, 1 (2009)

[35] See supplemental material for additional details of the calculations at the basis of the results presented in the main text.

[36] A. Puglisi, S. Pigolotti, L. Rondoni, and A. Vulpiani, Entropy production and coarse graining in Markov processes, Journal of Statistical Mechanics: Theory and Experiment 2010, P05015 (2010).

[37] B. Hughes, Random walks and random environments: random walks (1995).

[38] M. Esposito and K. Lindenberg, Continuous-time random walk for open systems: Fluctuation theorems and counting statistics, Physical Review E - Statistical, Nonlinear, and Soft Matter Physics 77, 10.1103/PhysRevE.77.051119 (2008).

[39] D. Andrieux and P. Gaspard, The fluctuation theorem for currents in semi-Markov processes, Journal of Statistical Mechanics: Theory and Experiment 2008, 10.1088/17425468/2008/11/P11007 (2008).

[40] D. T. Gillespie, Exact stochastic simulation of coupled chemical reactions, in Journal of Physical Chemistry (1977).

[41] G. Teza and A. L. Stella, In preparation.

[42] G. Teza, S. Iubini, M. Baiesi, A. L. Stella, and C. Vanderzande, Rate dependence of current and fluctuations in jump models with negative differential mobility, Physica A: Statistical Mechanics and its Applications 10.1016/j.physa.2019.123176 (2019), arXiv:1904.05241. 